\title{
A Model for Some Subcortical DTI Planar and Linear Anisotropy
}

\author{
Song Zhang and David Laidlaw
}

Brown University, Providence, RI 02912, USA

\section{Introduction}

Linear anisotropy, planar anisotropy and isotropy [1] are used as metrics for different kinds of diffusion in diffusion imaging. While linear anisotropy is reported to correlate to coherent neural fiber structures, the cause for planar anisotropy remains ambiguous. We hypothesize that overlapping linear structures and partial-volume averaging generate the planar anisotropy. We identify a subcortical region containing both linear and planar anisotropy in a human volumetric diffusion tensor image (DTI), propose a model of the anatomy and of the imaging process, and calculate simulated diffusion images of the anatomical model that qualitatively agree with the human DTI. Regions of planar anisotropy are common immediately beneath the cortex. Choosing one such region as representative, we model the anatomy with isotropic regions and linearly anisotropic structures. From the possibly overlapping model structures we simulate the diffusion imaging process, generating a series of diffusion weighted images (DWIs) of the anatomical structures. We then fit the DTI from these simulated DWIs and visualize it. The visualization agrees qualitatively with the visualization of a subcortical human DTI.

\section{Method}

We first identified an immediately subcortical region in the most superior part of the brain just adjacent to the interhemispheric fissure. Figure 1 shows a visualization of the white-matter structures in the region. The red streamtubes run along the direction of fastest diffusion in regions of linear anisotropy; the green surfaces show regions of planar anisotropy [2]. Our anatomical model comprises isotropic structures and linear structures. We represent isotropic regions like gray matter and fluid with isotropic diffusion tensors, and model linear structures with a cubic B-spline curve and a constant circular cross section. We then simulate DWIs from this anatomical model. In the simulation, we use isotropic tensors to represent isotropic structures; we use linear diffusion tensors whose major eigenvectors align with the tangent of the curve to represent linear structures. We generate DWIs from the anatomical model based on the relationship between the echo intensity and diffusion tensor given in [3].

To simulate the partial-volume effect, we supersample our anatomical model and then average the echo intensity over all the subsamples within one voxel. 


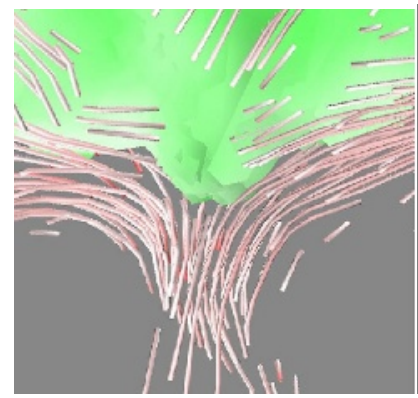

Fig. 1. In a subcortical region, complicated anisotropy patterns result in discontinuous fiber pathways. The dimensions of this feature are about $8 \times 9 \times 3 \mathrm{~mm}$. Image resolution is $1.70 \mathrm{~mm}$ isotropic

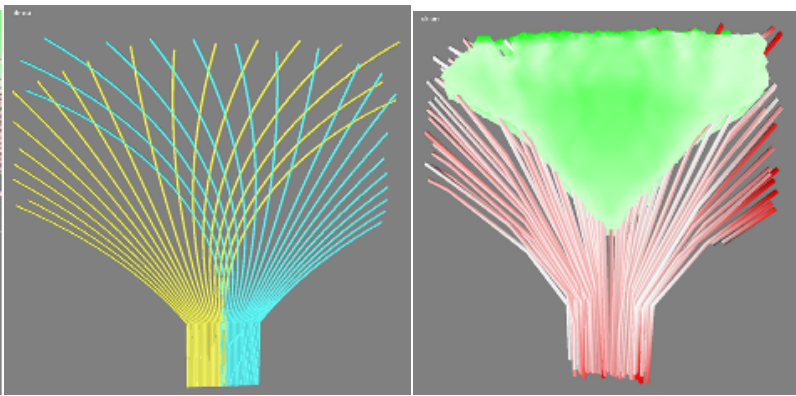

Fig. 2. We specify a qualitative model (shown here in 2D) with crossing fiber structures to represent the anatomy in Figure 1.
Fig. 3. A visualization of the DTI/DWIs generated from the anatomical model in Figure 2. Note that the planar anisotropy in the middle forms a pattern similar to Figure 1.

We constructed the phantom model shown in Figure 2 to be analogous to the region shown in Figure 1. We use the same visualization method that helped us identify the subcortical region of anisotropy to visually analyze our synthetic images.

\section{Results and Discussion}

Figure 1 shows a small subcortical region in which fibers emerge from the bottom, then splay out and cross each other, creating the planar anisotropy in the middle of the figure. Figure 2 shows our anatomical model. After synthesizing 25 DWIs (12 directions with b values of 500 and 1000 and a non-weighted diffusion image) from the model and fitting the DWIs to create a DTI, we visualize the DTI as in Figure 3. Note that the partial-volume effect in the region of crossing fibers creates planar anisotropy similar to that in Figure 1. The various crossing patterns and fiber densities result in various planar anisotropies, reflected by the different shades of green in the results. We also found that the streamtubes in the crossing area lie between the two crossing fibers. The result supports our hypothesis that overlapping structures and partial-volume averaging generate the planar anisotropy and also bias the direction of fastest diffusion in some regions of linear anisotropy away from the underlying fiber direction. Compensating for these distortions may be important in synthesizing accurate quantitative DTI analyses. 
Acknowledgments. Support from NSF CCR-0086065, the Human Brain Project (NIBIB and NIMH), NIMH, Alzheimer's Assoc, and the Ittleson Fund at Brown.

\section{References}

[1] Westin et al. ISMRM97

[2] Zhang et al. IEEE TVCG. 9:454-462(2003)

[3] Basser et al. J. Magn. Reson. B 103:247-254(1994) 